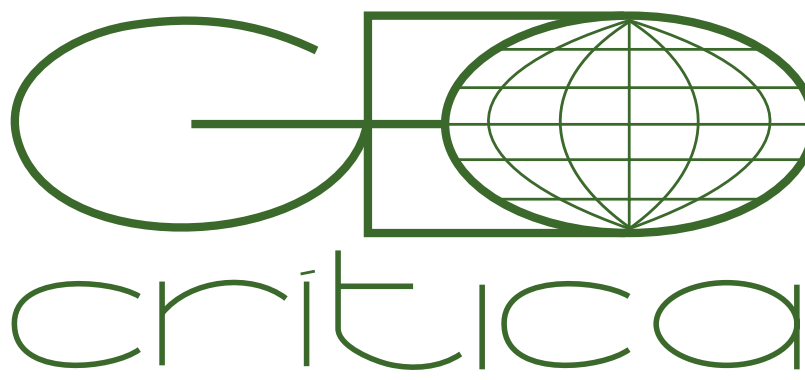

\section{Scripta Nova}

Revista Electrónica de Geografía y Ciencias Sociales Universitat de Barcelona

1 de junio de 2019

\title{
MEGAPROYECTOS EN EL ESPACIO AGRARIO DEL URUGUAY: EL AGRONEGOCIO DE LA SILVICULTURA
}

\begin{abstract}
Mauricio Ceroni
mceroni@ei.udelar.edu.uy

Recibido: 16 de febrero de 2018; Devuelto para correcciones: 21 de junio de 2018; Aceptado: 8 de diciembre de 2018

\section{Megaproyectos en el espacio agrario del Uruguay: el agronegocio de la silvicultura (Resumen)}

El crecimiento actual de los megaproyectos en el mundo es un rasgo central del funcionamiento de los espacios globales de acumulación de capital. Las corporaciones transnacionales son actores clave, tanto por la ejecución de la inversión como por la incidencia que realizan sobre los Estados. En este contexto, el presente escrito analiza, desde un abordaje crítico, el crecimiento de los megaproyectos agroindustriales del sector silvícola en el espacio agrario del Uruguay, ocurridos a comienzos del siglo XXI, como parte del modelo del agronegocio en el marco de la actual fase del capitalismo global. Para ello realicé una sistematización de las principales características de los megaproyectos, su origen, su expansión territorial y su incidencia sobre las comunidades locales. Finalmente, se muestra como el Estado está subordinado al dominio del capital transnacional, ya que tres corporaciones transnacionales concentran casi el $58 \%$ en superficie y un $84 \%$ en las exportaciones del sector.
\end{abstract}

Palabras clave: Megaproyectos, Agronegocio, Silvicultura, Estado, Uruguay

\section{Megaprojects in Uruguay agrarian space: the silviculture agribussines (Abstract)}

Nowadays the megaproject growth is a central characteristic of the capital accumulation global spaces. The key actors are the transnational corporations, which influence the States to set their projects. In this context, this paper analyzes, from a critical approach, the growth of the agro-industrial megaprojects of the forestry sector in the agrarian space of Uruguay, which occurred at the beginning of the 21st century, as part of the agribusiness model in the framework of the current phase of global capitalism. Therefore, I systematized the megaprojects principal characteristics, origin, territorial expansion, and impact in local communities. Finally, I conclude that the State is subordinated to the transnational capital domain, because almost $70 \%$ of the space and the $84 \%$ of the exportation belong to three transnational corporations.

Keywords: Megaprojects, Agribussines, Silviculture, State, Uruguay 
En la actual fase del capitalismo global se pueden identificar una serie de elementos como son; I) la conformación de una economía global, II) el surgimiento de una clase capitalista transnacional, III la existencia de nuevas relaciones de desigualdad, dominación y explotación en la sociedad, y IV) la re-configuración de un Estado transnacional ${ }^{1}$. Dichos elementos se pueden diferenciar de otras etapas del capitalismo, como fueron la mercantil en primera instancia, la industrial monopólica en segunda instancia y la industrial oligopólica en tercera instancia².

Un elemento transversal que toca los elementos mencionados, tiene que ver con el desarrollo de los "megaproyectos", siendo relevantes en la fase actual del capitalismo, ya que son grandes dinamizadores de producción de nuevos espacios del capital, reconfigurando la territorialidad existente.

El Uruguay no es ajeno a dicho proceso global, siendo la instalación de megaproyectos, especialmente en el sector de la silvicultura, un rasgo central que caracteriza el proceso de producción del espacio agrario. Debido a su despliegue espacial y a su impacto económico en la matriz productiva del país, el sector silvícola ha estado en foco de varias investigaciones en distintas áreas de conocimiento. En el área ambiental, se destacan estudios relacionados a los impactos que producen las grandes plantaciones de árboles, sobre la dinámica del agua y de los nutrientes del suelo, junto con el impacto que generan los plaguicidas a diversas especies de animales y vegetales nativos, entre otras varias investigaciones 3 . En relación a las ciencias sociales, se destacan los estudios desde la sociología, en donde analizan las implicancias y los efectos que ha generado el sector forestal en relación a la población local, el ambiente y las políticas establecidas por parte de los gobiernos de turno ${ }^{4}$. A su vez, también es importante mencionar el análisis del mercado laboral de la cadena forestal, asociado a las nuevas formas de la relación capital-trabajo ${ }^{5}$, sumado también al estudio del desarrollo de la infraestructura portuaria y la vinculación con el crecimiento del sector ${ }^{6}$. Desde la economía, existen estudios que se concentran en el empleo de metodologías, mediante la aplicación de diversos indicadores socioeconómicos ${ }^{7}$ y junto con el empleo del análisis costo-beneficio ${ }^{8}$, para evaluar el sobre el funcionamiento general del sector forestal sobre el conjunto de la economía del país, además de observar la organización y el funcionamiento de los grupos de inversión más relevantes ${ }^{9}$. Finalmente, en la Geografía, se destaca principalmente el estudio de Gautreau (2014) denominado "Forestación, territorio y ambiente, 25 años de silvicultura transnacional en Uruguay, Brasil y Argentina". Dicho autor, desde una perspectiva histórico-geográfica considerando cuestiones

\footnotetext{
1 Robinson 2015.

2 Wallerstein 1988.

3 Delgado, et al. 2006; Barreto 2008; Rubio 2012.

4 Ortiz, et al. 2005.

5 Carámbula y Piñeiro 2006; Carámbula, et al. 2013.

6 Piñeiro 2012.

7 Irisity y Chiappe 2002.

8 Morales y Siry 2009.

9 Mendell, et al. 2007.
} 
de poder y territorio, realiza una investigación muy profunda sobre el desarrollo histórico de la silvicultura en la región platina, cuyo interés transita por cuestionar el papel que establecieron los actores públicos y la sociedad para dotarse (o no) de herramientas que sirvan para regular el accionar de las empresas que comandan el sector silvícola.

En este sentido, la gran mayoría los trabajos mencionados abordan problemáticas de gran relevancia para el Uruguay ya que profundizan los impactos, a distintos niveles y dimensiones, que está generando el sector silvícola en el país. De igual forma, existe poco análisis crítico y profundo del funcionamiento general del sector, rescatando más la cuestión local y articulando de forma limitada con el funcionamiento del capitalismo global. En este marco, el presente escrito tiene como finalidad analizar el crecimiento de los megaproyectos agroindustriales del sector silvícola en el espacio agrario del Uruguay, ocurridos a comienzos del siglo XXI, como parte del modelo del agronegocio en el marco de la actual fase del capitalismo global. Dicho análisis se realizará desde una perspectiva crítica, tomando elementos de la geografía, la economía y la sociología, entendiendo a la crítica como "una manera de ver el mundo no como pura negatividad, sino como una vía de acceso a lo real que halla y fija los puntos fallidos de la dinámica totalizadora del capitalismo"10. De esta forma este trabajo es de los primeros en considerar que los megaproyectos.

Para ello, el trabajo se dividió en cuatro partes, en la primera se abordan cuestiones teóricas del modo de producción del capitalismo actual, profundizando los cuatro elementos que destaca Robinson (2015). En este contexto global, durante la en la segunda parte, analizaré una forma de organización específica del capital en el espacio agrario latinoamericano como es el "modelo del agronegocio" siendo un rasgo central para comprender la particularidad de los sectores transnacionales agrarios. En la tercera parte, pondré el foco en la dimensión espacial que adquiere el modelo del agronegocio, expresado mediante el desarrollo de los megaproyectos. Finalmente, en la cuarta parte me centraré en evidenciar de manera más empírica como se ha desarrollo del sector de la silvicultura Uruguaya, haciendo énfasis en el papel que desempeñó Estado Uruguayo para el crecimiento del sector.

\section{Elementos de la nueva fase actual del capitalismo La conformación de una economía global}

Lo primero que hay que comprender es que nos encontramos en una fase del capitalismo en donde la híper-conectividad del conjunto de las mercancías se hace cada día más profunda. La venta de la fuerza de trabajo, junto con el desarrollo de los medios de producción, ha generado que las partes que integran un objeto provengan de diversos puntos del globo. Este objeto transformado en mercancía en el mundo capitalista, genera una condensación de un conjunto de flujos, materia y energía, llegando a una intensidad tal, en donde la fetichización de la mercancía se profundiza, cada vez se hace más difícil discernir su composición.

10 Fuentes 2015, p. 98. 
Tal conectividad también se expresa en el flujo de los capitales, que permite una circulación muy intensa en el conjunto de la economía mundial, reorientando el peso de la economía nacional, ya que los componentes de lo "nacional" se insertan de manera distinta a la economía global de producción y finanzas, se pasa de una economía mundial en donde los Estados-Nación estaban vinculados entre sí a través de flujos comerciales y financieros a una economía global en la que el proceso de producción mismo está fuertemente integrado. Dicha movilidad le ha permitido al conjunto de capitales explorar en el mundo las mejores condiciones de rentabilidad para su instalación, los cuales buscan, reducir los costos de las materias primas y la fuerza de trabajo, y evadir las regulaciones de carácter impositivo por parte de los Estados-Nación junto con los controles ambientales ${ }^{11}$.

Este conjunto de condiciones que busca el capital lo realiza en base a una elevada cuota de explotación del trabajo, que genera altas ganancias y concentración de capital, estableciendo un sobrante de flujo monetario disponible para una nueva inversión, situación que se ve frenada en el sector productivo debido a las bajas tasas de rentabilidad, por tanto, este excedente de capital tiende a migrar hacia la esfera financiera, favorecido por la dicha conectividad, lo que permite que exista una circulación permanente e intensa del flujo de capitales ${ }^{12}$. Dicha superexplotación del trabajo, se expresa en un aumento sostenido de la precariedad laboral en el mundo, existiendo un aumento de las formas informales de trabajo, ya sea trabajadores por cuenta propia, familiares o asalariados sin contratos ${ }^{13}$. Estas nuevas formas de trabajo, pasan a ser muy importantes, no solo en la cantidad de personas que cada vez más se encuentran en esta situación, sino también replantea y abre un abanico de diversas formas de explotación, no tradicionales como el proletariado clásico.

\section{La conformación de una clase capitalista transnacional}

Otro rasgo central que se destaca en esta nueva fase del capitalismo, está íntimamente relacionado con los puntos que se describieron anteriormente, vinculado más como una consecuencia que va produciendo esta fase del capitalismo más que una causa, es lo que Robinson define como la conformación de una clase capitalista transnacional (CCT) ${ }^{14}$. En donde estaría integrada por las clases tradicionales de "explotadores" y "explotados" pero ahora de forma transnacionalizada, operando en los mercados y circuitos de acumulación global. Siguiendo al autor, el proceso de la globalización actúa de forma dual en términos subjetivos a la formación mundial de la clase. Por un lado, existiría una "fuerza centrípeta para la clase capitalista", en donde deben participar e integrar posiciones de mando en las cadenas globales de producción si quieren seguir siendo competitivos, lo que lleva a "una ampliación de horizontes subjetivos y de sensibilidad político-social". Por otro lado, actuaría una "fuerza centrífuga para la clase trabajadora y populares" quienes se enfrentan

\footnotetext{
11 Robinson 2015.

12 Rubio 2012.

13 OIT 2015.

14 Robinson 2015.
} 
a la competencia intensa de sus homólogos en todo el planeta, profundizando la fragmentación de la sociedad capitalista la cual genera un obstáculo para establecer "solidaridades intersubjetivas"15.

En base a una revisión de antecedentes el mismo Robinson (2015) rescata que existen ciertas características que van configurando a esta CCT, algunos de ellos son el fuerte aumento de la inversión extranjera directa, la expansión de las CTN, la concentración y fusión de capitales de diversas naciones, la creciente alianza entre empresas de punta, entre otras.

El aumento de la composición de la CCT mundial y su vínculo con la concentración de la riqueza se observa de manera elocuente. Si tomamos la riqueza total del planeta durante período 2010-2015, se observa lo siguiente, para el año 2010 el 1\% más rico de la población (388 personas) concentran la riqueza del 50\% de las personas más pobres del todo el planeta, lo que equivale a más de 3600 millones de personas, mientras que para el año 2015, el 1\% más rico de la población se concentraba en 62 personas ${ }^{16}$. Una forma que presentan los ricos de hacerse más ricos, es evadir cualquier tipo de impuestos que le puedan afectar su plusvalía, lo que ha llevado a los últimos años a desarrollarse de manera creciente los denominados "paraísos fiscales", en los cuales, esconden 7,6 billones de dólares de fortunas individuales, una cantidad superior al PIB del Reino Unido y Alemania juntos ${ }^{17}$. De las 62 personas la gran mayoría son las cabezas centrales de las corporaciones transnacionales globales, lo que da cuenta de que la mayor concentración de la riqueza global proviene de dichas corporaciones. Por tanto, si bien la economía global crece, la brecha entre ricos y pobres es una característica de esta nueva fase del capitalismo global.

\section{La existencia de nuevas relaciones de desigualdad, dominación y explotación en la sociedad}

El establecimiento y la profundización de la economía global asociada a la CCT han agudizado las relaciones de desigualdad, dominación y explotación del conjunto de la sociedad global. El carácter dual del capitalismo basado en la fragmentación a nivel local y la homogeneización a nivel global genera una agudización de las desigualdades sociales, limitando la cohesión social y el potencial revolucionario de la clase explotada. Los patrones clásicos de diferencia socioeconómica entre el centro y periferia ya se están materializando internamente en el centro, producto de un deterioro estructural que se observa en la actualidad, asociado en gran medida a la crisis global del capitalismo del año $2008^{18}$. La excesiva y exacerbada concentración de la riqueza de la CCT produce una fractura cada vez más intensa entre dicha clase y el resto de la sociedad global, existiendo un amento los mecanismos de sobrevivencia del las clases más bajas. De ahí que existe un aumento de las diversas formas de explotación, surgiendo con fuerza el trabajo informal e ilegal de la población

15 Ibid., p.50.

16 OXFAM 2016.

17 Ibid.

18 Robinson 2015. 
sobrante. No solo el aumento de la riqueza por parte de la CCT está generando dicha desigualdad, existen muchos otros factores más que responden al funcionamiento intrínseco del modo de producción capitalista. Vale mencionar uno muy importante el aumento de la robotización mundial, el cual está generando un incremento sostenido de la población sobrante (ejército industrial de reserva) no calificada, pero también calificada, lo que diferencia cualitativamente este período del capitalismo global de otros anteriores ${ }^{19}$.

\section{La re-configuración de un Estado transnacional}

El modo de producción capitalista global ha re-configurado el papel de los Estados-Nación, en donde los Estados con esencia neoliberal, son grandes actores que facilitan la acumulación del capital de las Corporaciones Transnacionales (CTN). Algunas medidas empleadas por los Estado que contribuyen al beneficio de las CTN son; i) "adoptar políticas fiscales y monetarias que garanticen la estabilidad macroeconómica."; ii) "proporcionar la infraestructura básica necesaria para la actividad económica global (puertos y aeropuertos, redes de comunicación, sistemas educativos, etc.)", iii ) "proveer el orden social, es decir, la estabilidad, lo que requiere instrumentos que mantengan el control social, aparatos coercitivos e ideológicos" ${ }^{20}$. Dichas medidas se observan de forma muy clara a lo largo y ancho de nuestro continente latinoamericano, en donde la inversión extranjera a cargo de las CTN parecería ser el objetivo de las economías nacionales, bajo el argumento que generan empleos y la economía crece. Estas son las medidas que el capitalismo, bajo su clase representante, nos intenta imponer de forma permanente separando la economía de la política, haciéndonos creer que la economía nacional se soluciona con la atracción de inversiones y que el "desarrollo" de nuestras sociedades depende de las potencialidades económicas ${ }^{21}$.

Otro elemento que caracteriza esta nueva configuración del Estado es la injerencia cada vez mayor de las instituciones transnacionales sobre el dictamen de las políticas de los Estados, los casos más paradigmáticos son el Fondo Monetario Internacional (FMI) y el Banco Mundial (BM) ordenando por dónde deben transitar los países en términos económicos. Pero no solo son estos organismos, sino que existen una infinidad de ellos, en el área del trabajo, la educación, la salud, la alimentación, etc. Dichas instituciones se entremezclan y se insertan en los aparatos del Estado conformando una compleja red de flujos que van incidiendo sobre las políticas nacionales. Esto genera que los Estados Nacionales de Latinoamérica cada vez tengan menor capacidad de armonizar los intereses sociales internos en conflicto, ya que la soberanía es cada vez menor. Este nuevo papel del Estado, establece que cada vez más el centro de poder de la acumulación se mueva de la clase burguesa nacional para la CCT, como se mencionó anteriormente ${ }^{22}$.

19 Mautz, et al. 2014.

20 Robinson 2015, p.53, 54.

21 Osoriom 2016.

22 Robinson 2015. 
Por último, el autor plantea cuales son los mecanismos que emplea el Estado de Estados Unidos de América (EUA) frente a los Estados-Nacionales, en donde destaca que su intervención es para el beneficio de los intereses de las CTN y de la CCT de origen estadounidense. Ya queda corta la teoría clásica de Lenin y en cierta medida de Luxemburgo sobre el imperialismo, ya no son los estados poderosos contra los estados más débiles, sino que son los Estados poderosos que penetran en otros Estados para generar el marco necesario del desembarco de las CTN ${ }^{23}$. Una evidencia clara de tal fenómeno es el modelo del agronegocio, que sirvió de camino para la instalación de las CNT agrarias estadounidenses en el espacio agrario latinoamericano.

\section{El modelo del agronegocio}

Una de las formas productivas que modificó ciertas relaciones de producción en el espacio agrario latinoamericano, fue el modelo del agronegocio. El concepto de agronegocio proviene de la Escuela de Administración y Negocios de la Universidad de Harvard, en EUA, y se explicita en el libro "A Concept of Agribusiness" de John Davis y Ray Goldberg publicado en el año 1957, en donde definen al "Agribusiness ${ }^{24 "}$ (...) "al total de operaciones que integran; la producción de manufacturas y su distribución; la producción de productos en las fincas; el almacenamiento, procesamiento y distribución de las "commodities" agrícolas y artículos producidos a partir de ellas"25

El libro parte de la premisa central que el campo estaría pasando por profundas transformaciones productivas en base a la "revolución tecnológica" proveniente de los avances de la segunda guerra mundial, teniendo como base el "progreso" científico utilizado para la agricultura, siendo la fórmula para solucionar los desajustes y desequilibrios en un proceso evolutivo ${ }^{26}$. Según los autores, el principal cambio que ocurre en las fincas es que dejarían de ser de autosubsistencia para pasar a ser de función comercial, con una producción basada en los monocultivos. Actividades como el almacenamiento, procesamiento y distribución de los productos deberían ser transferidas para otras empresas, que pasarían a producir productos industriales, como tractores, camiones, combustibles, fertilizantes, agroquímicos, entre otros ${ }^{27}$.

La influencia de John Davis y Ray Goldberg traspasa el ámbito académico y se disemina en los tomadores de decisión de la política agrícola de EUA. Es así que durante la década del 60 y 70 se va expandir el modelo del agronegocio por latinoamérica, llegando a ser en la actualidad una forma de organización productiva que rige a los territorios agrarios principalmente del cono sur. Dicha expansión tuvo diversos mecanismos, el primero siendo la más importante fue el ámbito de la política de "lobby", que establecía EUA, en donde buscaba "convencer" a los gobiernos

23 Ibid.

24 La traducción a la lengua española es agronegocio. Desde ahora en adelante en el texto se hará referencia a "modelo de agronegocio".

25 Davis y Goldberg 1957, p.2.

26 Ibid.

27 Ibid. 
de los países latinoamericanos, con ayuda de las elites burguesas agrarias nacionales que integraban parcial o totalmente los gobiernos de la época, de los beneficios económicos productivos del modelo. Este mecanismo se iba materializando con la modificación y elaboración de nuevos instrumentos jurídicos mediante leyes o decretos para facilitar la entrada de los capitales extranjeros. Un segundo ámbito está relacionado con el financiero, ya que los organismos regionales e internacionales, mediante el consentimiento de los EUA, realizaban los préstamos financieros para implementar el modelo en los diversos países. Un tercer ámbito, se vincula con el científico-tecnológico, consistió en que los países receptores adopten los diversos paquetes tecnológicos elaborados por EUA y sean insertados en las raíces de los sistemas productivos. Esto iba acompañado de apoyo financiero para desarrollo científico local, en donde el paquete tecnológico tuviera mejor adaptabilidad, para lograr aumentar la productividad agronómica y por ende la ganancia de los capitales extranjeros. Dentro de este ámbito se desarrolló la denominada "revolución verde" la cual consistió en la introducción de "variedades de alto rendimiento" principalmente trigo y arroz junto con la aplicación de un paquete de fertilizantes y agroquímicos para sostener esa mejora de los cultivos ${ }^{28}$. El objetivo era aumentar la productividad agronómica y obtener mayores cantidades de materias primas agrarias para aumentar las ganancias de las empresas de EUA con el discurso de disminuir el problema de hambre y alimentación del planeta ${ }^{29}$.

Este proceso que fue tejiendo el imperio norteamericano, se materializó mediante una de sus formas más elocuentes, la expansión de sus capitales por el mundo, en este caso el desembarco de capitales en diversas ramas de la economía latinoamericana. Para el año 1945 existen 182 subsidiarias americanas en latinoamérica, para el año 1955 pasaron a ser 357 y para el año 1965 eran 888 empresas, lo que implicó un desembarco de más de 2.m741 millones de dólares al año $1965^{30}$

En la década de los 80 y 90 este modelo adquiere desde el punto de vista tecnológico una nueva fase, la "segunda revolución verde" basada en el desarrollo y la difusión de la biotecnología ${ }^{31}$, siendo la transgénesis uno de los avances más emblemáticos. La transgénesis consiste en la utilización de los sistemas biológicos y organismos vivos como elementos de manipulación genética para poder crear nuevos organismos, en otras palabras son distintos fragmentos de ADN originados por diferentes organismos, ensamblados en un único gen ${ }^{32}$.

Según Taddei (2013) basado en Porto Gonçalves, (2006), esta última revolución verde ha traído una separación entre el saber y el hacer, ya que la innovación y producción del conocimiento en laboratorios de punta, impulsada por las empresas transnacionales las cuales privatizan el conocimiento mediante la propiedad intelectual, genera una dependencia aún mayor de los productores agropecuarios, prin-

28 Griffin 1982.

29 Taddei 2013.

30 Cueva 1979.

31 Taddei 2013.

32 Onofre 2002. 
cipalmente de los pequeños, “...expropiando y mercantilizando los saberes agrícolas y obligándolos a adoptar un patrón productivo cada vez más estandarizado"33

Finalmente, este modelo se basa en una mayor acumulación de capital, basado en un elemento central que se diferencia de otros períodos del capitalismo agrario, que es el "acaparamiento de tierras". Si bien esta última estrategia no es exclusiva de latinoamérica, si no que ocurre en otras partes del mundo como en África subsahariana, presenta un diferencial, ya que el acaparamiento no es solo extra-regional sino que también es intraregional, en manos de empresas (trans) latinoamericanas, basadas en estrategias de extranjerización y alta concentración de la tierra ${ }^{34}$. Este tipo de empresas tienen un vínculo directo con el exterior, pero su vez, en muchos casos ocurre que los capitales son regionales, como ocurre principalmente en Bolivia, Paraguay y Uruguay mediante la llegada de capitales argentinos o brasileños ${ }^{35}$. Esta situación intraregional, pone en evidencia que la región no es homogénea y que si bien se diferencia de otras regiones planetarias existe una dinámica interna que está operando de forma permanente y que repercute en los distintos países.

Dichas empresas, buscan una expansión intra-regional basada principalmente en la disminución de los riesgos que ponen en jaque la acumulación de capital. En este sentido, buscan una diversificación, tanto biofísica (clima, suelos), como también en políticas institucionales vinculada el régimen monetario y/o financiero. Esto las hace que tengan prácticas de intervención política en órdenes espaciales que trascienden los Estados-Nación. Un ejemplo de esta estrategia es como las empresas transnacionales del sector de la silvicultura, analizan de forma global las ventajas comparativas de una conjunto de países, entre ellos Argentina, Brasil y Uruguay ${ }^{36}$.

\section{La producción del espacio de los megaproyectos}

Para avanzar en el análisis y comprender cómo se va tejiendo y produciendo el espacio en base las prácticas políticas que realizan las empresas transnacionales, es necesario definir en primera instancia que entendiendo por espacio. Siguiendo a Lefebvre (2013) concibo al espacio como la manera en que "envuelve a las cosas producidas y comprende sus relaciones en su coexistencia y simultaneidad: en su orden y/o desorden (relativos)"37. Esas relaciones son relaciones sociales y están generadas por acciones que "unas remiten al universo de la producción, otras al del consumo"38. A su vez el autor agrega que el espacio "se reorganiza en función de la búsqueda de recursos cada vez más escasos: energías, agua, luz y algunas materias primas de origen vegetal o animal"39, en donde su unidad de análisis se integra por "las fuerzas productivas y sus componentes (naturaleza, trabajo, técnica y conoci-

33 Taddei 2013, p.160.

34 Borras Jr, et al. 2012.

35 FAO 2011.

36 Cubbage, et al. 2010.

37 Lefebvre 2013, p.129.

38 Ibid., p.129.

39 Ibid., p.383. 
miento), las estructuras (relaciones de propiedad), las superestructuras (las instituciones y el mismo Estado)" 40 . Por tanto "el capitalismo se sirve de todas las abstracciones, de todas las formas, incluida la ficción jurídica y legal de la propiedad, de todo cuanto parecía irreductible en principio a la apropiación privada o privativa (la naturaleza, la tierra, las energías vitales, los deseos y las necesidades )"41.

Esta abstracción en la cual se sustenta el capitalismo, le sirve al capital para desarrollar megaproyectos que se establecen principalmente en la esfera productiva y que generan una reorganización al espacio.

El término "megaproyecto" está directamente vinculado a la construcción de infraestructura, siendo esta un elemento central del modo de producción capitalista tanto para la acumulación como la reproducción del capital, que va de la mano del desarrollo de las fuerzas productivas. Se lo podría definir como "un tipo de producción espacial que obedece a la única demanda del capitalismo la producción de capital, la cual se despliega hoy en día a escala global. La realización [...] requiere los oficios de la esfera política, así como los instrumentos financieros del gran capital, basados en la vanguardia del desarrollo científico-técnico"42. No son elementos nuevos en la fase actual del capitalismo sino que han adquirido un peso y una dimensión central para la reproducción ampliada del capital.

El modo de producción capitalista se ha enfrentado en las últimas décadas a crisis más recurrentes que integran una crisis estructural la cual se mantiene desde la década de los 80 del siglo pasado y está basada en una constante caída de la tasa de ganancia del capital global ${ }^{43}$. El aumento de la frecuencia de las crisis capitalistas, principalmente las económicas y financieras, establece que una forma de salida de la misma sea por intermedio de la destrucción de capital fijo y creación de nuevos fijos, de ahí que el capital busque las “fijaciones/soluciones provisionales espacio-temporales (fix)" 44 para remendar la crisis, pero dichas soluciones no atacan el problema de fondo que es revertir la tasa global de ganancia.

Una materialidad concreta de las fijaciones es la producción de megaproyectos, los cuales por su gran condensación de capital son infraestructuras centrales en la actualidad del capitalismo. Según Ibarra (2016) y en base a la interpretación de su escrito puedo identificar cinco características centrales que presentan los megaproyectos; i) es un tipo de producción espacial en la actual fase del capitalismo siendo la máxima expresión del espacio tanto desde el poder como desde su vertiente racionalista e instrumental; ii) se producen en espacios rurales como urbanos y su desarrollo moviliza gran cantidad de recursos financieros, materiales, científico-técnicos y de población en general; iii) su desarrollo incluye las etapas de producción, circulación y consumo dentro del capitalismo; iv) son espacios de convergencia de diversas escalas espaciales, ya en su gran mayoría son capitales transnacionales; v)

40 Ibid., p.141.

41 Ibid., p.383.

42 Ibarra 2016, p.39,40.

43 Sotelo 2014.

44 Harvey 2014, p.154. 
su construcción genera resistencias sociales y son catalizadores de surgimientos de movimientos y organizaciones sociales.

En definitiva la fase actual del desarrollo del capitalismo global se sustenta principalmente en una economía de carácter global que se articula en base a un Estado que cada vez más adquiere comportamientos transnacionales, comandados por una clase capitalista no tradicional. En función de esta triada es que se va produciendo un espacio transnacional organizado, en este caso, mediante el modelo del agronegocio que se materializa y se evidencia tácitamente mediante la instalación de los megaproyectos que buscan recursos cada vez más escasos, como son las materias primas de origen vegetal.

Para evidenciar dicho proceso, analizaré el desarrollo de la silvicultura en el Uruguay, en donde destacaría como la instalación de los megaproyectos está vinculada directamente al impulso del Estado, lo cual ha generado la producción de un espacio sin precedentes en la historia del país.

\section{Los megaproyectos de la silvicultura en Uruguay}

A finales del año 2017 se cumplieron 30 años de promulgación de la primera ley que sentó las bases para desarrollo del sector silvícola en el Uruguay. Se puede dividir al desarrollo del sector en dos etapas, la primera entre 1988-2005, la cual forjó las bases del modelo forestal en el Uruguay, consistió en una serie de medidas legislativas que tenían como objetivo impulsar un sector incipiente en el país. Se crea la ley marco ( 15.939) en donde se destacan, la prohibición de la tala del bosque nativo; el incentivo de proyectos para la plantación de de bosques artificiales, con criterios de localización (suelos de prioridad forestal), y a su vez, el estimuló a inversores de otros sectores de la economía (nacional o extranjera) a invertir en forestación y en industrias madereras a través de subsidios que van desde la fase de producción, en relación a las plantaciones y tenencia de la tierra, la fase de cosecha en la exoneración de impuestos a la maquinaria y en el estímulo a la baja de la tasa de interés para préstamos financieros ${ }^{45}$. En definitiva fueron subsidios sobre el recurso tierra y sobre el capital, estableciendo el marco para la acumulación de capital del conjunto de empresas que invirtieron en el sector. A dicha ley se le sumaron los beneficios de la ley 16.906, la cual tenía como objetivo promover la inversión en el conjunto de la economía nacional, estableciendo un mecanismo jurídico denominado "proyectos declarados de interés nacional" en donde los proyectos que cumplían con determinadas condiciones, se les exoneraba impuestos referidos al patrimonio, a la renta y a la importación de bienes de capital, entre otros ${ }^{46}$.

Estos mecanismos se vieron reflejados en un aumento sostenido de la superficie plantada, principalmente de Pinos y Eucaliptus, pasando de 10.000 hectáreas plantadas en el año 1990 a 85.000 hectáreas en el año 1998, decreciendo de forma

45 Ley 15.9391987.

46 Ley 16.9061988. 
sostenida al año 2002 a menos de 20.000 hectáreas $^{47}$. Existiendo un acumulado de 970.000 hectáreas al año $2007^{48}$.

Cuadro 1. Superficie de las plantaciones silvícolas para el año 2011, pertenecientes a los megaproyectos de UPM, Montes del Plata y BTG Pascual por región para la totalidad del país.

\begin{tabular}{lllllll}
\hline $\begin{array}{l}\text { Regiones } \\
\text { Silvícolas }\end{array}$ & $\begin{array}{l}\text { Superficie } \\
\text { Silvícola } \\
\text { Total } \\
(\%)\end{array}$ & $\begin{array}{l}\text { Superficie } \\
\text { Silvícola } \\
\text { UPM } \\
(\%)\end{array}$ & $\begin{array}{l}\text { Superficie } \\
\text { Silvícola } \\
\text { Montes del } \\
\text { Plata } \\
(\%)\end{array}$ & $\begin{array}{l}\text { Superficie } \\
\text { Silvícola } \\
\text { BTG } \\
\text { Pascual } \\
\text { (Weyer- } \\
\text { haeuser) } \\
(\%)\end{array}$ & $\begin{array}{l}\text { Superficie } \\
\text { Silvícola } \\
\text { BTG } \\
\text { Pascual } \\
(\%)\end{array}$ & $\begin{array}{l}\text { Superficie } \\
\text { Silvícola } \\
\text { Total } \\
\text { CTN (\%) }\end{array}$ \\
\hline Centro-Este & 12.3 & 0 & 3.5 & 4.6 & 0.0 & 8.1 \\
Oeste & 35.9 & 21.4 & 11.9 & 0.0 & 0.0 & 33.3 \\
Noreste & 23.2 & 4.7 & 0.5 & 8.1 & 0.0 & 13.3 \\
Sureste & 22.2 & 0 & 0.0 & 0.0 & 3.4 & 3.4 \\
Total Regiones & 93.6 & 26.1 & 15.9 & 12.7 & 3.4 & 58.1 \\
Resto País & 6.4 & 0.0 & 0.0 & 0.0 & 0.0 & 0.0 \\
\hline Total País & $\mathbf{1 0 0}$ & $\mathbf{2 6 . 1}$ & $\mathbf{1 5 . 9}$ & $\mathbf{1 2 . 7}$ & $\mathbf{3 . 4}$ & $\mathbf{5 8 . 1}$ \\
\hline
\end{tabular}

Fuente: DINOT/FAO (2011), Gautreau (2014), Informes técnicos de las empresas disponibles en las páginas webs (véase bibliografía). Nota: La construcción de la tabla se basó en la información que brindan las CTN de su ubicación de las plantaciones por departamento, lo que permitió regionalizar para tener una mejora distribución de donde se concentran las plantaciones.

La segunda etapa, se extiende desde el año 2006 hasta el presente, la cual se caracteriza por una retracción del papel del Estado, que se materializa con la eliminación de los subsidios que inician en el año 2002 mediante la ley 17.453 y terminan en el año 2005 con la ley 17.905 que determina la supresión definitiva de los apoyos fiscales al sector de la silvicultura ${ }^{49}$.

Posteriormente, mediante una serie de decretos se ajustan los suelos de prioridad forestal redireccionando la plantación de las regiones silvícolas. Dichas medidas tuvieron efectos inmediatos, bajando sensiblemente las plantaciones por debajo de las 10.000 hectáreas el año 2010 y manteniendo la superficie total del país en 990.000 hectáreas para el año $2012^{50}$. En la actualidad, es el tercer sector en importancia de la agropecuaria del Uruguay, por detrás de la agricultura y la ganadería.

Un elemento central que estructura el sector es referido a su composición de capitales, siendo en su gran mayoría CTN de diferentes fracciones de capital, de las

47 Gautreau 2014.

48 DIEA 2011.

49 Ley 17.453 2002; Ley 17.9052005.

50 DIEA 2016. 


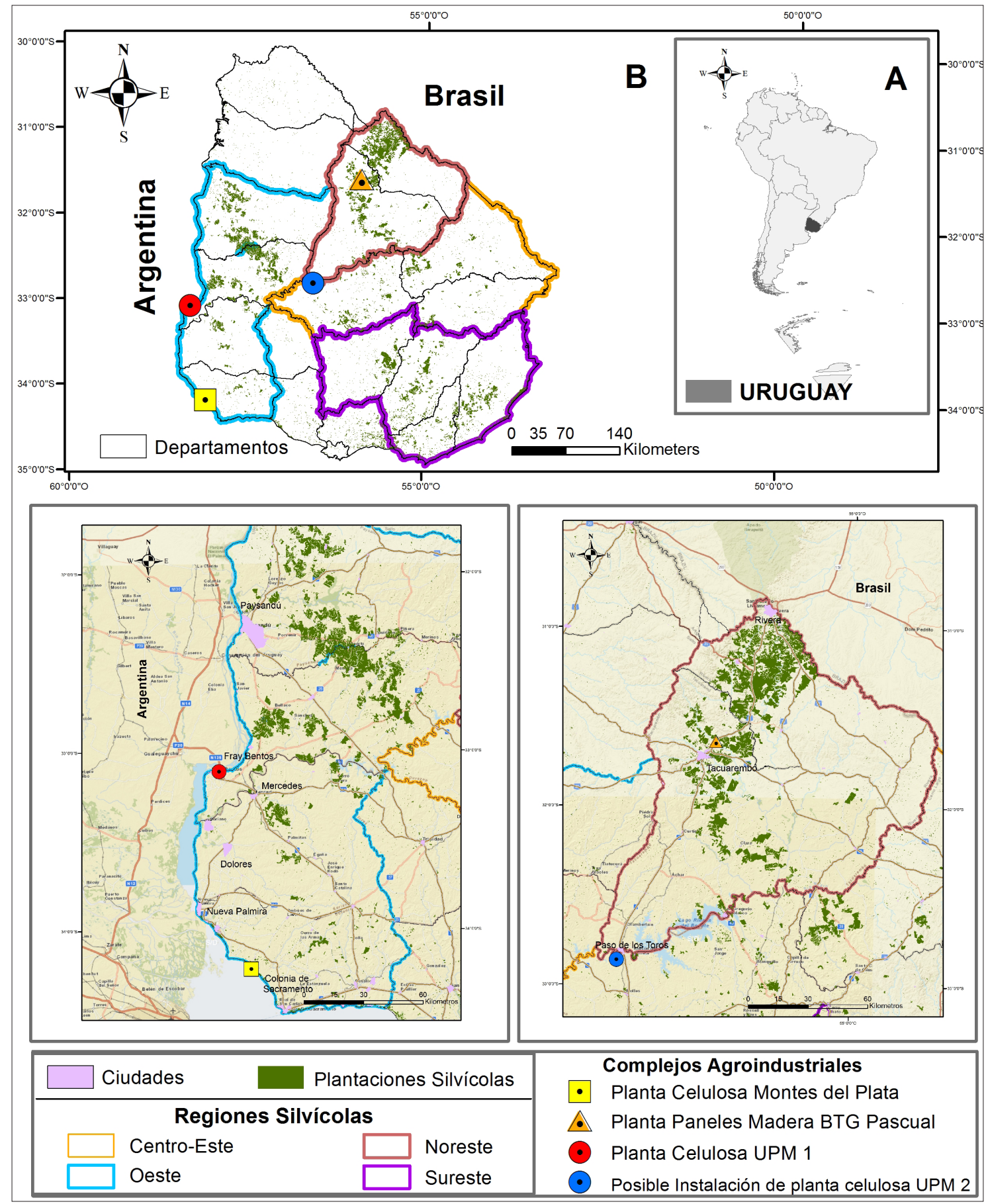

Figura 1: A. Uruguay en el contexto de América del Sur. B. Principales regiones silvícolas en el Uruguay. C. Distribución espacial de las plantaciones silvícolas y los complejos agroindustriales de las CTN en la región oeste de Uruguay para el año 2011. D. Distribución espacial de las plantaciones silvícolas y los complejos agroindustriales de las CTN en la región noreste de Uruguay para el año 2011.

Fuente: Elaboración propia a partir de DINOT/FAO (2011)

cuales se han ido concentrando y expandiendo a lo largo de los últimos años, siendo en la actualidad un sector oligopólico en el cual tres empresas controlan el $84 \%$ de las exportaciones totales del sector para el año $2016^{51}$ y abarcan un 58,1\% de la 51 Uuguay XXI 2016. 
superficie total de las plantaciones silvícolas (cuadro 1). Observando el cuadro 1 y la figura 1 se aprecia que los megaproyectos se concentran en diversas zonas del país.

Finalmente, una estrategia que tienen las propias CTN es el diseño de políticas que apunten a una ideologización del territorio, sobre la incidencia de las comunidades rurales que potencialmente pueden ser afectadas por la instalación del megaproyecto. En este sentido, crean mecanismos institucionales como son las Fundaciones de Responsabilidad Empresarial (FRE) en el cual cumplen un doble propósito, por un lado su legitimidad para el desarrollo del proyecto y por otro lado, la exoneración de ciertos tributos que permiten contribuir a las ganancias de las empresas.

Para comprender mejor cómo se estructura el sector vamos a mencionar algunas características de los megaproyectos de cada una de las CTN.

\section{UPM-Kymmene}

Dicha CTN es una corporación transnacional de origen finlandesa conformada por la fusión de Kymmene Corporation y Repola Ltd (subsidiaria United Paper Mills Ltd) en el año 1996. En la actualidad tiene presencia en 13 países reuniendo a unos 19.300 empleados y sus ventas anuales ascienden a cerca de 10 mil millones de Euros. Su principal producto es la pulpa de celulosa para la generación de papeles de distinta calidad, pero también se muestra como una empresa productora de Bioenergía ${ }^{52}$. Para el año 2011 era la décimo segunda empresa más importante del sector en el mundo ${ }^{53}$.

Llega al Uruguay en el año 2009 con la compra de la totalidad del paquete de activos de la empresa "Metsä-Botnia" por unos 2.400 millones de dólares, la cual operaba en Uruguay desde comienzos de 1990. Dentro de la compra se adquiere la industria de producción de celulosa y unas 180.000 hectáreas $^{54}$. En la actualidad cuenta con una producción de 1.300.000 toneladas anuales de pulpa de celulosa y posee unas 215.000 hectáreas bajo su gestión ${ }^{55}$. Su producción de concentra en la región Oeste del país cercana la al complejo agroindustrial de Fray Bentos (cuadro 1 y figura 1).

El rol del Estado fue decisivo para su instalación, no solo por los beneficios fiscales que le otorgó, como fue la producción industrial en régimen de zonas francas, la exoneración de importaciones de bienes y equipos de maquinaria, la eliminación del impuesto a patrimonio, la compra de energía por parte del Estado en 40 megawatts (MW), entre otros, sino que en base a la amenaza del diferendo diplomático con la república Argentina y su posterior resolución en la corte internacional de la Haya $^{56}$, se creó una especie de "nacionalismo" en base a la defensa de Uruguay debido a su seriedad y responsabilidad en el control ambiental, que tenía por detrás la defensa de la transnacional. La resolución internacional fortaleció al Estado urugua-

52 UPM 2018.

53 Gautreau 2014.

54 Espectador 2009.

55 UPM Uruguay 2017.

56 ICJ 2015. 
yo y a la CTN. En la actualidad el poder ejecutivo negocia un nuevo megaproyecto que traerá otra nueva planta de producción de pasta de celulosa sobre los márgenes del Rió Negro, la cual se maneja una inversión de 4000 mil millones de dólares y el Estado se compromete a invertir 1000 millones $^{57}$. Recientemente se firmó un acuerdo marco en el ámbito laboral suscrito por, el Estado, las patronales empresariales (cámara de la construcción y la cámara metalúrgica) y la central sindical (PIT-CNT) para establecer las bases de la relación capital-trabajo y así establecer un "entorno propicio para la potencial inversión de UPM en el Uruguay"58. Además se firmó un contrato de viabilidad de la inversión, comprometiendo una serie de recursos económicos y ambientales, dejando en evidencia como el propio Estado se somete a los intereses de las CTN.

Finalmente, la CTN creó una fundación llamada "fundación UPM" la cual se define en su página web como promotora de “...el desarrollo de las comunidades donde la empresa opera a través de la educación, la capacitación y el emprendedurismo, fomentando una cultura de vida saludable ${ }^{\prime \prime 59}$. Presenta una incidencia territorial en más de 130 comunidades rurales del país, financiando proyectos vinculados principalmente hacia la educación (primaria, secundaria y terciaria), cuya relación principal es el vínculo con la capacitación laboral basada en una visión emprendedora. Desarrolla proyectos en Uruguay desde hace 10 años, en los cuales ha transitado diversas temáticas como es la promoción del deporte, la conservación del ambiente, la salud odontológica, el fortalecimiento de bibliotecas comunitarias, entre otras ${ }^{60}$.

\section{Montes del Plata}

La corporación transnacional "Montes del Plata" es una asociación estratégica denominada "Joint Ventures" entre distintos capitales, en el cual asumen el riesgo de la inversión de forma compartida. Dicha asociación ocurre muchas veces donde existe una empresa ya instalada a nivel nacional y la empresa transnacional quiere instalarse de forma tal que su inversión sea rentable. En este caso el consorcio de capitales pertenecen a "Arauco" empresa de origen Chilena ${ }^{61}$ dedicada a la producción de celulosa y madera aserrada en América Latina (Chile, Argentina, Brasil) ${ }^{62}$, siendo la tercera empresa más importante del mundo en el sector para el año $2011^{63}$. Por otro lado, el capital "Stora Enso" empresa de origen Sueco-Finesa dedicada a la fabricación de paneles, cartones y productos forestales, empresa que opera en más de 35

57 Presidencia 2016.

58 Presidencia 2017.

59 Fundación UPM 2017.

60 Ibid.

61 En la actualidad es muy difícil identificar el origen del país en la composición de los capitales, ya que los mecanismos que ha desarrollado el capitalismo financiero a escala global son muy diversos, de ahí que la mayoría de las CTN estén integradas por fracciones de capital de diversos orígenes.

62 Montes del Plata 2017.

63 Gautreau 2014. 
países en el mundo ${ }^{64}$, se ubicaba para el año 2011 en el puesto número ocho dentro del sector mundial forestal ${ }^{65}$.

En el Uruguay la empresa se consolida en el año 2009, luego de adquirir la mayoría del paquete que estaba en manos de la empresa "ENCE", que incluía la mayor parte de las plantaciones forestales, un puerto privado (M'Bopicuá) y un terreno para la construcción de la agroindustria ${ }^{66}$. En la actualidad el megaproyecto de "Montes del Plata" abarca en la fase productiva unas 145.000 hectáreas, la fase industrial, la producción de pasta de celulosa de 1.300.000 toneladas anuales junto con la generación de energía eléctrica para la producción y un excedente, de alrededor de $80 \mathrm{MW}$ de potencia, y la fase comercial, mediante una terminal portuaria sobre el Rió de la Plata que le permite exportar al mundo la mercancía producida. La inversión estimada en el complejo fue de 2.500 millones de dólares ${ }^{67}$. Se estima que el total de los activos de la corporación en Uruguay es de 3.500 millones de dólares ${ }^{68}$. La producción se concentra en mayor medida en el Oeste, pero también presenta producción en la región Centro-Oeste y Noreste del país (cuadro l y figura 1).

También para este caso el papel del Estado fue determinante para que se instalará en Uruguay, principalmente en la construcción del complejo industrial, firmando un contrato de confidencialidad, en el cual se mantenía bajo reserva las negociaciones que dicho Estado realizaba con la empresa. Este contrato fue inconstitucional y luego de un recurso jurídico se dio a conocer. Dentro de los elementos que el Estado se comprometió fue establecer la declaración de inversión de "interés nacional" publicado en el diario oficial para la exoneración de impuestos (patrimonio) durante el proceso de construcción del megaproyecto, la exoneración de tributos en la fusión de las diversas empresas que tiene el grupo inversor, el cambio de categoría de suelos cercanos a la industria para establecer beneficios en las autorizaciones ambientales, facilitar el dragado del Rio de la Plata y otorgarle las condiciones de navegabilidad a sus embarcaciones, respetar los convenios de inversiones que Uruguay tiene entre Filandria y Chile y darle las garantías futuras a la empresa para presentar demanda en el Centro Internacional de Arreglo de Diferencias Relativas a Inversiones (CIADI ${ }^{69}$. Por último hay que destacar que su excedente energético se lo vende a la empresa eléctrica del Estado Uruguayo por lo que la empresa obtiene una ganancia en la producción de energía y el Estado está obligado a comprarla.

También hay que destacar el papel que juega la CTN en relación a su incidencia territorial. En este sentido, la empresa no tiene una fundación directa como UPM pero si dentro de su estructura corporativa se muestra como una empresa que presenta una estrategia con la comunidad social la cual, “...apunta a gestionar los impactos de nuestras actividades, a fin de contribuir al desarrollo local, a la mejora de

64 Stora Enso 2017.

65 Gautreau 2014.

66 Pou 2016.

67 Montes del Plata 2017.

68 Presidencia 2014.

69 Judicial 2012. 
la calidad de vida, generando instancias de escucha y diálogo en las comunidades donde opera. Involucrando distintas alianzas estratégicas entre el sector público, privado y el tercer sector, como un ejemplo de integración de los diferentes actores que componen la comunidad bajo un objetivo común de desarrollo social sostenible"70. En este sentido, apunta a un relacionamiento con la comunidad que involucra a asociaciones de pequeños productores, escuelas rurales con énfasis en la educación ambiental, apoyo a diversas policlínicas y la promoción en educación y seguridad vial. Por último hace un fuerte hincapié en la promoción de la conservación del ambiente, gestionado una reserva de fauna y flora (Bioparque $M^{\prime}$ Bopicuá ${ }^{71}$.

\section{BTG Pactual Timberland Investment Group}

Finalmente, el fondo de inversión "BTG Pactual Timberland Investment Group" cuya sede principal se encuentra en San Pablo y en Nueva York, pero se integra de capitales de diversas partes del mundo. Es una empresa de inversión en diversos rubros y su objetivo es mejorar la rentabilidad de la inversión de sus capitales. No es una empresa exclusiva de producción forestal como los casos anteriores. Recientemente compró la totalidad del paquete accionario de la empresa "Weyerhaeuser" de origen estadounidense, la cual se caracteriza por ser una corporación transnacional destinada a la plantación de bosques artificiales, para la elaboración paneles de compensado de distinto tipo de madera, que se usan en la industria de la construcción y en la industria del transporte, para la elaboración de diversos productos ${ }^{72}$. Dicha empresa presenta operaciones en más de 8 países y para el año 2011 se encontraba en el puesto 11 de las empresas más importantes del sector en el mundo ${ }^{73}{ }^{19}$ y se había instalado en Uruguay desde el año 1996, siendo su primer país de Sudamérica en establecer inversiones. En el año 2006 dio inicio el proceso de industrialización, creando una planta destinada a la producción de compensado para paneles de madera $^{74}$. La compra de del grupo inversor "BTG Pactual" por 402.5 millones dólares incluye unas 120.000 hectáreas y la planta de producción de paneles de madera ${ }^{75}$. El grupo inversor ya tenía presencia en Uruguay desde el año 2013 y posee en la actualidad unas 32.000 hectáreas $^{76}$ con dicha compra aumenta su patrimonio en tierra a unas 152.000 hectáreas. En mayor medida las plantaciones que pertenecían a "Weyerhaeuser" se localizan en la región Noreste y parte en la región Centro-Este. Mientras que las plantaciones que pertenecen a "BTG Pactual" se concentran en su totalidad en la región Sureste del país (cuadro 1 y figura 1).

El Estado uruguayo tuvo un papel activo, no tanto como los casos anteriores, pero sí favoreció la instalación de la industria, ya que también exonera de impuestos

70 Montes del Plata 2017.

71 Montes del Plata 2017.

72 Weyerhaeuser 2017a.

73 Gautreau 2014.

74 Weyerhaeuser 2015

75 Weyerhaeuser 2017b

76 BTG Pactual 2017 
en la construcción de la planta, la cual produce alrededor de 500 toneladas de tableros por día. Además de dicha exoneración la empresa también genera una planta de energía en base a sus desechos de biomasa, aportando unos $12 \mathrm{MW}$ que son comprados por la empresa estatal.

En relación al desarrollo de políticas vinculadas a las comunidades rurales, el grupo "BTG Pactual" desde su presencia en Uruguay no presenta explícitamente programas de apoyo a las comunidades. La empresa "Weyerhaeuser" si financiaba proyectos vinculados a la RSE, a través de distintas ONGs, no presentaba una fundación explícita como los casos anteriores, sino más bien su incidencia era por intermedio de apoyo económico a diversas organizaciones en la zona. Sus objetivos eran "colaborar y apoyar el desarrollo de las comunidades, focalizándose en las áreas de influencia en donde opera la empresa"77. Dentro de los proyectos financiados se encuentran los vinculados a la educación ambiental por intermedio de las escuelas rurales, capacitación laboral en la formación terciaria y actividades relaciones con el sector salud y seguridad ${ }^{78}$.

\section{Conclusiones}

El abordaje crítico desarrollado durante este artículo, considerando elementos de la geografía, la economía y la sociología permitió demostrar cómo la organización del sector silvícola Uruguayo es un claro ejemplo del funcionamiento del capitalismo global actual.

Los planteamientos desarrollados por Robinson (2015) brindaron un marco potente para analizar el comportamiento de un sector en relación al Estado y al funcionamiento del capitalismo global. En este sentido, destacó como el modelo del agronegocio impulsado a mediados del siglo XX fue un mecanismo potente que determinó la injerencia de las CTN en el espacio agrario latinoamericano, sustentada por el apoyo de los organismos multilaterales. Esta composición de carácter transnacional re-configuró el rol de Estado frente a la penetración masiva de capitales extranjeros. En la actualidad, el Estado Uruguayo ha recorrido la senda en búsqueda del incremento constante de la inversión extranjera directa, estableciendo instituciones que se encargan de promover e impulsar la atracción de capitales, brindando una serie de beneficios fiscales ${ }^{79} 57$.

Dicha política se le suma a la baja densidad de población rural y la nula resistencia por parte de las organizaciones sociales, otorgándoles a los capitales demasiadas garantías para la inversión. Un elemento que faltaría para configurarse un Estado con características completas de carácter transnacional está vinculado más con la inversión en el desarrollo de infraestructura básica para garantizar una mejora circulación de las mercancías. Según los que comandan las políticas económicas de nuestros países, como son los organismos internacionales, una de las limitantes que

77 El Observador 2014

78 Ibid.

79 Uruguay XXI 2017. 
enfrenta el país es superar el atraso en el desarrollo de infraestructura, para seguir por la senda del crecimiento económico, ininterrumpido desde hace 14 años $^{80}$. La fuerte influencia de las CTN en la economía nacional muestra cómo los países, y en este caso el Uruguay, van reconfigurando su economía de base mundial a una organización más de economía global, en donde las exportaciones de las principales mercancías las comandan las CTN. Este elemento evidencia como cada día más las relaciones comerciales están mediadas y matrizadas por las CNT y no por la clase burguesa nacional como ocurría en el siglo pasado. Sería interesante a futuro analizar el vínculo de la CCT con la clase capitalista nacional.

Este comportamiento actual del capital en el cual me centre en los párrafos genera una producción del espacio agrario basada en megaproyectos principalmente de carácter productivo, evidenciando de forma clara lo que plantea Ibarra (2016) sobre las características centrales que establecen los megaproyectos. La única condición que no identifique fue la vinculada a la resistencia social. Si bien existen organizaciones sociales que se oponen al desarrollo de los megaproyectos, como "REDES-AT" y "Uruguay libre contra la megaminería" no existe un despojo directo sobre comunidades indígenas o campesinos, ya que por un lado no existen como tales, sino que tienen otra nomenclatura como es la "Agricultura Familiar" ${ }^{81} y$ por otro lado, la historia de conformación de la estructura agraria se basa en la propiedad privada, por tanto, los megaproyectos compran sus tierras bajo este régimen y desarrollan sus proyectos sin ningún problema. Este elemento se suma al desarrollo de estrategias que realizan las CTN en su fase ideológica, ya que es un sector con gran inversión sobre los mecanismos de incidencia territorial, esto puede estar asociado al funcionamiento propio del sector, ya que en su fase productiva existe un tiempo como mínimo de ochos años antes de la primera cosecha, por tanto, para que no se afecte el funcionamiento productivo, se debe tejer relaciones de legitimidad y buena visibilidad con los pobladores locales, para evitar cualquier conflicto que dañe la inversión de capital inicial.

En definitiva la producción del espacio del capital transnacional agrario adquiere un poder creciente y a veces inimaginable, frente al propio Estado y al conjunto de las clases sociales nacionales que pierden soberanía política y económica, y sobre todo, convenciendo y limitando otras formas de producir riqueza social.

\section{Bibliografía}

BARRETO, P. Efectos iníciales de la forestación sobre la calidad del agua de escurrimiento en una cuenca del Río Tacuarembó. Tesis (Maestría en Ciencias Agrarias) Universidad de la República, 2008.

BID. Estratégia país 2015-2020. Washington 2015.

80 BID 2015.

81 Klein 2007. 
BORRAS JR, S. M., J. C. FRANCO, S. GÓMEZ, C. KAY, et al. Land grabbing in Latin America and the Caribbean. Journal of Peasant Studies, 2012, 39(3-4), 845872.

BTG PACTUAL. BTG Pactual Timberland Investment Group. 15 septiembre 2017. Disponible en: https://www.btgpactual.com/home_en/AssetManagement. aspx/Timberland

CARÁMBULA, M., S. FIGUEREDO y M. BIANCO Resolviendo las necesidades del capital: del intermediario laboral a la empresa de servicios agrícolas. Revista de Ciencias Sociales, 2013, 26(32), 35-52.

CARÁMBULA, M. y D. PIÑEIRO La forestación en Uruguay: Cambio demográfico y empleo en tres localidades. Agrociencia, 2006, 10(2), 63-73.

CUBBAGE, F., S. KOESBANDANA, P. MAC DONAGH, R. RUBILAR, et al. Global timber investments, wood costs, regulation, and risk. Biomass and Bioenergy, 2010, 34(12), 1667-1678.

CUEVA, A. El desarrollo del capitalismo en América latina. Ciudad de México: Siglo XXI, 1979.

DAVIS, J. y R. GOLDBERG A Concept of agribusiness. Boston: Harvard University Graduate School of Business Administration, 1957.

DELGADO, S., F. ALLIAUME, F. GARCÍA PRÉCHAC y J. HERNÁNDEZ Efecto de las plantaciones de Eucalyptus sp. sobre el recurso suelo en Uruguay. Agrociencia, 2006, 10(2), 95-107.

DIEA. Anuario 2010. In D.E. AGROPECUARIAS. Montevideo: Ministerio de Ganadería Agricultura y Pesca (MGAP), 2011.

DIEA. Anuario 2015. In D.E. AGROPECUARIAS. Montevideo: Ministerio de Ganadería Agricultura y Pesca (MGAP), 2016.

DINOT/FAO Atlas de Cobertura de Suelo de Uruguay: Cobertura del Suelo y Detección de Cambios 2000-201 1. Land cover classification System. Montevideo: OPP/MGAP/MVOTMA/FAO/UNESCO, 2011.

EL OBSERVADOR. Suplemento Weyerhaeuser 10 agosto 2017. Disponible en: https://issuu.com/elobservador.com.uy/docs/weyerhaeuser_2014

ESPECTADOR. Economía. 19 de Agosto 2017. Disponible en: http://www.espectador. com/economia/ 165 196/grupo-upm-compro-planta-de-botnia-y-planeaexpandirse-mundialmente-en-el-negocio-de-celulosa

FAO. El Acaparamiento de Tierras en América Latina y el Caribe Visto Desde una Perspectiva Internacional más Amplia. 2011.

FUENTES, D. Crítica de la razón histórica. En R. SERRUR ed. Bolívar Echeverría modernidad y resistencias. Ciudad de México: Ediciones ERA/Universidad Autónoma Metropolitana (UAM), 2015, p. 91-98. 
FUNDACIÓN UPM. Fundación UPM: 10 años en el Uruguay profundo. 29 agosto 2017. Disponible en: http://www.upm.uy/fundacion/Pages/Default.aspx

GAUTREAU, P. Forestación, territorio y ambiente: 25 años de silvicultura transnacional en Uruguay, Brasil y Argentina. Montevideo: Ediciones Trilce, 2014.

GRIFFIN, K. La economía política del cambio agrario. Ciudad de México: Fondo de Cultura Económica, 1982.

HARVEY, D. Diecisiete contradicciones y el fin del capitalismo Quito: Editorial IAEN, 2014.

IBARRA, M. V. Los megaproyectos desde una geografía crítica. En M.V. IBARRA y E. TALLEDOS eds. Megaproyectos en México una lectura crítica. Ciudad de México: Itaca, 2016, p. 21-42.

ICJ. Causa relativa a las plantas de celulosa en el Rió Uruguay (Argentina contra Uruguay). La Haya: 2015.

IRISITY, F. y M. CHIAPPE Indicadores socioeconómicos para la evaluación de la sustentabilidad forestal en el norte de Uruguay. Agrociencia, 2002, 16(1), 177-185.

JUDICIAL, R. Sentencia N56. In F.L.E.L. CIVIL. Colonia: Suprema Corte de Justicia, 2012.

KLEIN, F. El destino de los indígenas del Uruguay. Nómadas. Revista crítica de ciencias sociales y jurídicas, 2007, 1(15), 1-10.

LEFEBVRE, H. La producción del Espacio. Madrid: Capitán Swing Libros, 2013.

LEY 15.939. Ley Forestal. In M.D.G.A.Y. PESCA. Montevideo: Diario Oficial, 1987.

LEY 16.906. Ley de Promoción de Inversiones. In M.D.E.Y. FINANZAS. Montevideo: Diario Oficial, 1988.

LEY 17.453. Ley de Ajuste Fiscal. In M.D.E.Y. FINANZAS. Montevideo: Diario Oficial, 2002.

LEY 17.905. Ley de Regulación del sector Forestal. In M.D.G.A.Y. PESCA. Montevideo: Diario Oficial, 2005.

MAUTZ, F., J.CADEMARTORI y J. CADEMARTORI La humanidad sobrante: Una indagación sobre el desempleo. Santiago: Universidad de Santiago de Chile, 2014.

MENDELL, B., V. MORALES, Z. BENNADJI, A. MORENO, et al. Financing Uruguay's forestry sector: survey and case study. Journal of Forestry, 2007, 105(3), 125-130.

MONTES DEL PLATA. Bio Parque M’Bopicuá. 14 septiembre 2017. Disponible en: http://www.montesdelplata.com.uy/bioparque/index.php

MONTES DEL PLATA. La empresa. 10 septiembre 2017. Disponible en: https://www. montesdelplata.com.uy/nuestra-empresa-2 
MONTES DEL PLATA. Sustentabilidad. 10 septiembre 2017. Disponible en: https:// www.montesdelplata.com.uy/sustentabilidad_comunidad.php?lang=es

MORALES, V. y J. SIRY Economic impact evaluation of Uruguay forest sector development policy. Journal of Forestry, 2009, 107(2), 63-68.

OIT. Perspectivas sociales y Empleo del Mundo: Resumen. Ginebra: 2015.

ONOFRE, R. Impactos de OGMs en el medio ambiente y la agricultura. En A. CARMEN ed. Los transgénicos en la agricultura y la alimentación. Montevideo: UdelaR, 2002, p. 87-96.

ORTIZ, M. S., J. TAKS, B. SCHMID y S. THIMMEL Entre el desierto verde y el país productivo: El modelo forestal en Uruguay y el cono sur. Montevideo: Casa Bertolt Brech y REDES-AT, 2005.

OSORIO, J. Estado, reproducción del capital y lucha de clases. Ciudad de México: Universidad Nacional Autónoma de México, Instituto de Investigaciones Económicas, 2016.

OXFAM. Una economía al servicio del $1 \%$ [online]. [Oxford]: 2016. Disponible en $<$ WWW.oxfam.org $>$.

PIÑEIRO, D. Formas de resistencia de la agricultura familiar. El caso del Noreste de Canelones. Universidad de la República, 1985.

PIÑEIRO, D. Repercusiones de las inversiones forestales: la ampliación del puerto de la Paloma. Montevideo: Universidad de la República/Comisión Sectorial de Investigación Científica, 2012.

POU, R. Forestación en Uruguay: Una apuesta al porvenir. Montevideo: Ediciones Plus-Ultra, 2016.

PRESIDENCIA. Segunda planta de celulosa instalada en Uruguay sostiene 6.000 empleos y califica personal.9 julio 2017.Disponible en: https://www.presidencia. gub.uy/comunicacion/comunicacionnoticias/montes-plata-kreimermanempleos-califica-personal

PRESIDENCIA, D. L. R. Uruguay se prepara para recibir inversión de 5.000 millones de dólares, la más importante de su historia. 9 de julio 2017. Disponible en: https:// www.presidencia.gub.uy/comunicacion/comunicacionnoticias/conferenciavazquez-planta-upm

PRESIDENCIA, D. L. R. Gobierno avanza en negociaciones con UPM. In. Montevideo: Presidencia de la República, 2017.

ROBINSON, W. América Latina y el capitalismo global. Una perspectiva crítica de la globalización. Ciudad de México: Editorial Siglo XXI, 2015.

RUBIO, B. Explotados y excluidos: los campesinos latinoamericanos en la fase agroexportadora neoliberal. DF, México: Plaza y Valdés, 2012.

SOTELO, A. México (re)cardado. Dependencia, neoliberalismo y crisis. Ciudad de México: UNAM/Ítaca, 2014. 
STORAENSO. Sobre nosotros. 10 agosto 2017. Disponible en: http://www.storaenso. $\mathrm{com} /$

TADDEI, E. El agronegocio: de la república de la soja a los desiertos verdes. En J. SEOANE, E. TADDEI y C. ALGRANTI eds. Extractivismo, despojo y crisis climática. Desafíos para los movimientos sociales y los proyectos emancipatorios de nuestra América. Buenos Aires: Ediciones Herramienta, Editorial El colectivo yGEAL, 2013, p. 157-182.

UPM. Sobre Nosotros. 12 de agosto 2018. Disponible en: http://www.upm.com/ About-us/Pages/default.aspx

UPM URUGUAY. Inicio. 10 de agosto 2017. Disponible en: http://www.upm.uy

URUGUAY XXI. Exportaciones por Empresas, series históricas. In I.U.XXI. Montevideo: Instituto Uruguay XXI, 2016.

URUGUAY XXI. ¿Por qué Uruguay? Montevideo: 2017.

WALLERSTEIN, I. El capitalismo histórico. Ciudad de México: Editorial Siglo XXI, 1988.

WEYERHAEUSER. LUMIN. Montevideo: 2015.

WEYERHAEUSER. Timberlands. 29 agosto 2017. Disponible en: https://www. weyerhaeuser.com/

WEYERHAEUSER. Weyerhaeuser completes sale of uruguay timberlands and manufacturing business to a consortium led by btg pactual's timberland investment group 18 septiembre 2017. Disponible en: http://investor. weyerhaeuser.com/2017-09-01-Weyerhaeuser-completes-sale-of-Uruguaytimberlands-and-manufacturing-business-to-a-consortium-led-by-BTGPactuals-Timberland-Investment-Group

(c) Copyright: Mauricio Ceroni, 2019

(c) Copyright: Scripta Nova, 2019.

Ficha bibliográfica:

CERONI, Mauricio. Megaproyectos en el espacio agrario del Uruguay: el agronegocio de la silvicultura. Scripta Nova. Revista Electrónica de Geografía y Ciencias Sociales. Barcelona: Universidad de Barcelona, 1 de junio de 2019, vol. XXIII, nº 615. [ISSN: 1138-9788] 